Ebenhoch R, Saenger K, Crosa F, Gonzalez Y, Degen De

ARTÍCULO ORIGINAL

Arrua R. Plantas utilizadas en la medicina popular

ORIGINAL ARTICLE

paraguaya para tratar afecciones relacionadas al estrés.

Rev. Soc. cient. Parag. 2021;26(1):82-90.

https://doi.org/10.32480/rscp.2021.26.1.82

Recibido: 3/04/2021. Aceptado: 17/05/2021.

\title{
Plantas utilizadas en la medicina popular paraguaya para tratar afecciones relacionadas al estrés
}

\section{The plant species that are used in Paraguayan folk medicine to treat stress-related conditions}

\author{
Ruben Ebenhoch $^{1}$ (D), Karen Saenger ${ }^{1}$ (D), Fiorella Crosa ${ }^{1}$ (D), Yenny \\ Gonzalez $^{1}$ (i), Rosa Degen De Arrua ${ }^{1 *}$ id \\ ${ }^{1}$ Universidad Nacional de Asunción, Facultad de Ciencias Químicas, \\ Dirección de Investigaciones, Departamento de Botánica. San Lorenzo, \\ Paraguay.
}

Autor correspondiente: degenrosa@gmail.com

Resumen: La medicina tradicional ha mantenido su popularidad en todo el mundo. En Paraguay, la medicina tradicional es ampliamente practicada por sus habitantes para tratar las diversas afecciones que se les presenten, empleando principalmente plantas medicinales. Este trabajo tuvo como objetivo identificar las especies vegetales que se utilizan en la medicina popular paraguaya para tratar afecciones relacionadas al estrés. El estudio fue observacional; consistió en consultar a vendedores de plantas medicinales sobre aquellas que recomiendan para contrarrestar el estrés y sus síntomas, de 3 puestos de venta de "yuyos" de cada mercado situado en las ciudades de Limpio, San Lorenzo y Villa Elisa, Departamento Central. Se realizaron tres recolecciones entre julio y noviembre del 2019. Las plantas adquiridas fueron identificadas, fotografiadas, secadas, etiquetadas y depositadas en el Herbario FCQ. Como resultado se identificaron 16 especies, de las cuales 8 especies fueron recomendadas (en estado fresco) en el mercado de Limpio, 10 especies (6 especies en estado fresco y 4 especies en polvo grueso en estado seco dispuesto en bolsita) en Villa Elisa y 10 especies (en estado fresco) en San Lorenzo. Las especies citadas en los tres mercados son "menta'i", Mentha x piperita; "kapi'i katî", Kyllinga vaginata y "cedrón Paraguay", Aloysia citriodora. La familia más representada fue Lamiaceae, con tres especies, seguida de Verbenaceae con dos especies. 
Palabras clave: estrés, medicina popular, plantas medicinales.

\begin{abstract}
Traditional medicine has maintained its popularity worldwide. In Paraguay, practitioners of traditional medicine are abundant and formulate treatments based mainly on plants. This work aimed to identify the plant species that are used in Paraguayan folk medicine to treat stress-related conditions. The study was observational; It consisted in consulting sellers about the plants they recommend to counteract stress and its symptoms, from 3 "yuyos" stalls in each market located in the cities of Limpio, San Lorenzo and Villa Elisa, Central Department. Three collections were made between July and November 2019. The acquired plants were identified, photographed, dried, labeled and deposited in the Herbarium FCQ. As a result, 16 species were identified, of which 8 species were recommended (in the fresh state) in the Limpio market, 10 species ( 6 species in the fresh state and 4 species in coarse powder in the dry state arranged in a bag) in Villa Elisa and 10 species (fresh) in San Lorenzo. The species mentioned in the three markets are "menta'i", Mentha x piperita; "Kapi'i katĩ", Kyllinga vaginata and "Paraguay cedar", Aloysia citriodora. The most represented family was Lamiaceae, with three species, followed by Verbenaceae with two species.
\end{abstract}

Keywords: stress, folk medicine, medicinal plants.

\title{
1. INTRODUCCIÓN
}

La medicina tradicional ha mantenido su popularidad en todo el mundo. A partir de la década del 1990 se ha constatado un resurgimiento de su utilización en muchos países desarrollados y en desarrollo(1). Según OMS(2), el uso de las plantas medicinales constituye una terapia más natural, más inocua, efectiva, de un costo racional y accesible a las poblaciones. Además, se ha demostrado que la fitoterapia es el tratamiento alternativo y complementario más comúnmente utilizado(3). Entre los usos terapeuticos mas representativos se encuentran los digestivos y estomacales, hepatoprotector, para problemas de la piel, antisepticos, refrescantes y antipruritoso $^{(4)}$.

Según un estudio realizado en Perú, la acupuntura y la fitoterapia son los métodos más conocidos y aceptados de la medicina complementaria, siendo el uso de plantas medicinales el más resaltantes de la medicina popular(5). En Argentina se afirma de que los conocimientos sobre la medicina tradicional se adquiere de generación en generación como así también a 
través de libros especializados en el tema empleando numerosas plantas como parte del tratamiento de sus dolencias(4). En cuanto al Brasil, la practica está ampliamente extendida, especialmente empleando medicina tradicional China(6). Esta realidad no es ajena al ámbito Latinoamericano y por supuesto al Paraguay(7). En Paraguay los practicantes de la medicina tradicional son abundantes $y$ formulan tratamientos basados mayoritariamente en plantas ${ }^{(8)}$. y varios estudios han documentado su uso para el tratamiento de la ansiedad e insomnio( ${ }^{(9-12)}$. Actualmente el estrés y sus síntomas(13), ansiedad, insomnio, decaimiento, nerviosismo entre otros, se han convertido en uno de los principales problemas para la salud y la seguridad laboral(14). Este trabajo tuvo como objetivo identificar las especies vegetales que se utilizan en la medicina popular paraguaya para tratar afecciones relacionadas al estrés.

\section{MATERIALES Y MÉTODOS}

Se realizó un estudio observacional, que consistió en una breve entrevista con preguntas abiertas a los vendedores, consultándoles sobre las plantas medicinales que recomiendan para contrarrestar el estrés y sus síntomas, como también su forma de uso, según sus conocimientos populares.

Las preguntas y la adquisición de las plantas fueron hechas simulando ser compradores habituales en 3 puestos de venta de hierbas medicinales (yuyos) de cada mercado situados en las ciudades de Limpio, San Lorenzo y Villa Elisa, respectivamente, del Departamento Central, Paraguay.

Se realizaron tres recolecciones en total. Dos de ellas entre los meses de julio y agosto del año 2019, y otra en el mes de febrero del año 2020.

Las plantas adquiridas fueron identificadas, fotografiadas, secadas, envasadas en sobres, etiquetadas, dispuestas en cajas y depositadas en la colección de plantas medicinales en el Herbario FCQ. En las etiquetas se colocan los siguientes datos, nombre común, nombre científico, la fecha de compra, el mercado, se indica que el material se encuentra estéril y se señala "Proyecto plantas medicinales para el estrés" (Figura 1 y 2). 
Rev. Soc. cient. Parag. 2021;26(1):82-90

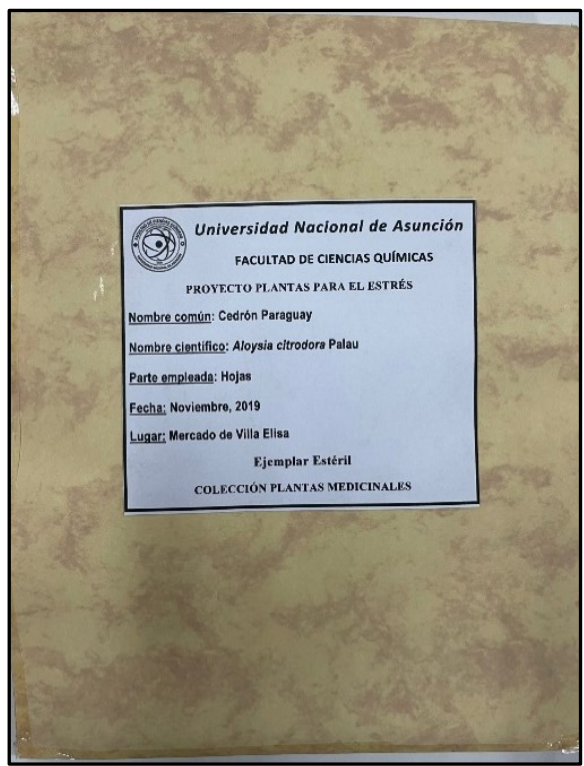

Figura 1. Cedrón Paraguay. Etiqueta.

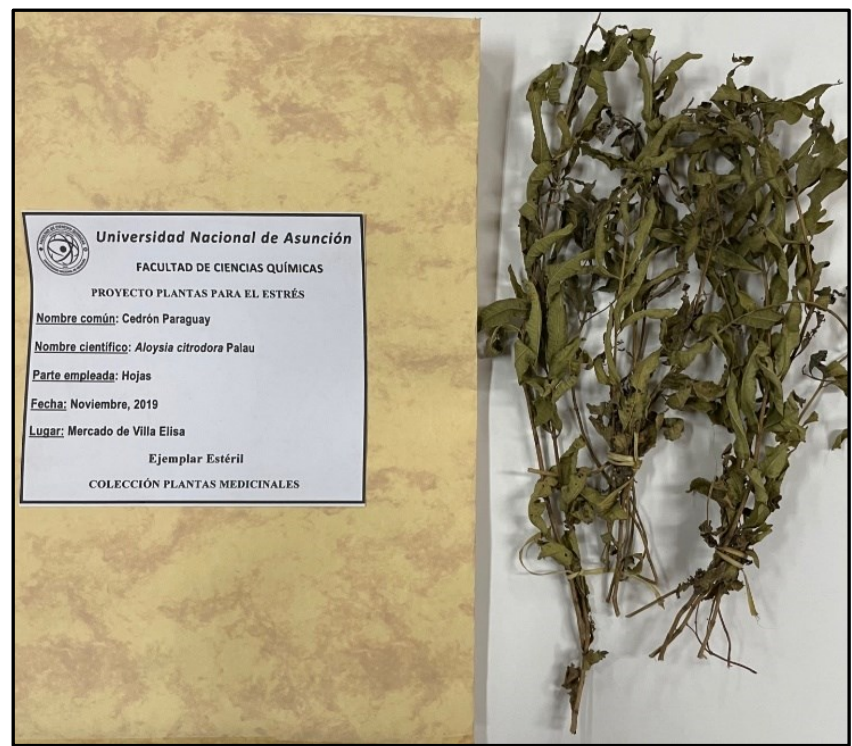

Figura 2. Planta medicinal que se conservará en sobre y con la etiqueta.

Ebenhoch R, Saenger K, Crosa F, Gonzalez Y, Degen De Arrua R. Plantas utilizadas en la medicina popular paraguaya para tratar afecciones relacionadas al estrés 


\section{RESULTADOS}

De los resultados de las entrevistas se elaboró una tabla donde se aclaran los nombres comunes y científicos junto con las partes utilizadas de las plantas.

Las plantas más recomendadas para tratar estas afecciones son Menta'i (Mentha x piperita), Cedrón Paraguay (Aloisya citrodora Paláu) y Kapi'i Kati (Killinga vaginata Lam.). La familia más representada fue Lamiaceae, con tres especies, seguida de Verbenaceae con dos especies.

Tabla 1. Las especies comercializadas que se recomiendan para contrarrestar el estrés y sus síntomas.

\begin{tabular}{|c|c|c|c|c|}
\hline Lugar & $\begin{array}{l}\text { Nombre } \\
\text { Común }\end{array}$ & Nombre Científico & Familia & Parte Usada \\
\hline \multirow[t]{11}{*}{ Limpio } & Agrial & Begonia cucullata & Begoniaceae & Tallo y hojas \\
\hline & Alfalfa & Medicago sativa & Fabaceae & Hojas \\
\hline & $\begin{array}{l}\text { Cedrón } \\
\text { Paraguay }\end{array}$ & Aloysia citrodora Paláu & Verbenaceae & Hojas \\
\hline & Hinojo & $\begin{array}{l}\text { Foeniculum vulgare } \\
\text { Mill. }\end{array}$ & Apiaceae & Semillas \\
\hline & Kapi'i kati & Kyllinga vaginata Lam. & Cyperaceae & Raíz \\
\hline & Manzanilla & Chamaemelum nobile & Asteraceae & Inflorescencias \\
\hline & Mbokaja'i & $\begin{array}{l}\text { Acrocomia aculeata } \\
\text { (Jacq.) Lodd. ex Mart. }\end{array}$ & Arecaceae & Raíz \\
\hline & Menta'i & Mentha $\times$ piperita & Lamiaceae & Hojas \\
\hline & Perdurilla & Gomphrena decumbens & Amaranthaceae & $\begin{array}{l}\text { Hojas, Tallo y } \\
\text { Raíz }\end{array}$ \\
\hline & Tarope'i & $\begin{array}{l}\text { Dorstenia brasiliensis } \\
\text { Lam. }\end{array}$ & Moraceae & Hojas \\
\hline & Valeriana & Valeriana officinalis $L$. & Valerianaceae & Raíz \\
\hline \multirow[t]{6}{*}{$\begin{array}{l}\text { San } \\
\text { Lorenzo }\end{array}$} & Burrito & $\begin{array}{l}\text { Aloysia polystachya } \\
\text { (Griseb.) Moldenke }\end{array}$ & Verbenaceae & Hojas \\
\hline & $\begin{array}{l}\text { Cedrón } \\
\text { Paraguay }\end{array}$ & Aloysia citrodora Paláu & Verbenaceae & Hojas \\
\hline & Hinojo & $\begin{array}{l}\text { Foeniculum } \\
\text { Mill. }\end{array}$ & Apiaceae & Semillas \\
\hline & Kapi'i kati & Kyllinga vaginata Lam. & Сyperaceae & Raíz \\
\hline & Menta'i & Mentha x piperita & Lamiaceae & Hojas \\
\hline & Romero & Rosmarinus officinalis $L$. & Lamiaceae & Hojas \\
\hline
\end{tabular}

Ebenhoch R, Saenger K, Crosa F, Gonzalez Y, Degen De Arrua R. Plantas utilizadas en la medicina popular paraguaya para tratar afecciones relacionadas al estrés 
Rev. Soc. cient. Parag. 2021;26(1):82-90

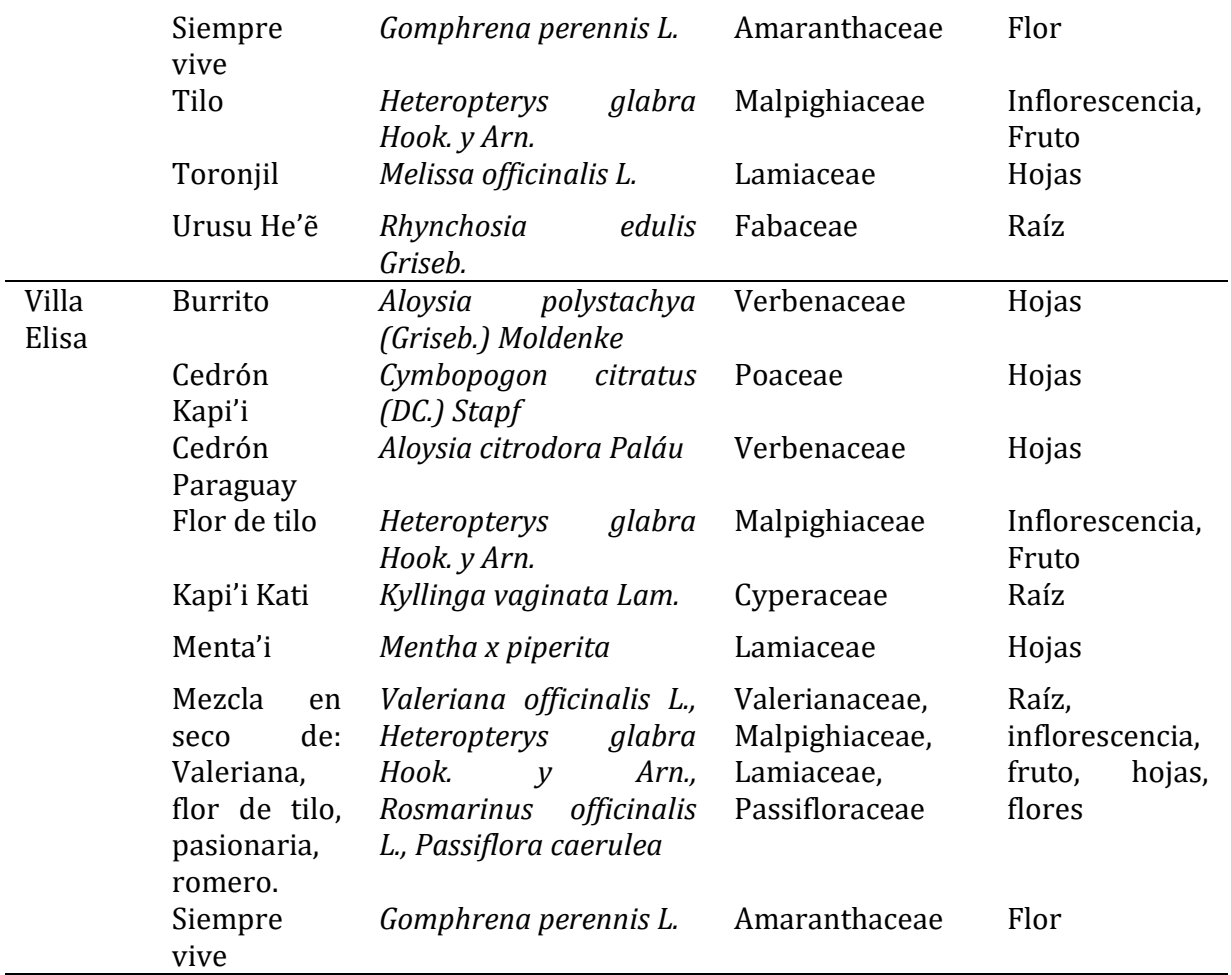

\section{DISCUSIÓN}

Se observa una predominancia en la recomendación de 3 plantas, de las cuales de la Menta'i (Mentha x piperita) se sabe que posee efectos relajantes gastrointestinales ${ }^{(15)}$ y es utilizada para reducir el dolor y ansiedad por medio de aromaterapia(16), también existe actividad reportada sobre el cedrón Paraguay (Aloysia citriodora Paláu) describiéndose su capacidad como relajante nervioso(17) y efecto sedativo (18); no obstante no se ha encontrado información en la literatura que apoye la utilización del Kapi'i Kati (Killinga vaginata Lam.) en el tratamiento del estrés y sus síntomas. Se puede observar que la mayoría de las plantas recomendadas por los vendedores son utilizadas también para tratar malestares digestivos, pudiendo así establecer que la medicina tradicional frecuentemente relaciona el estrés con síntomas digestivos ${ }^{(19)}$. Se necesitaría un estudio más amplio para determinar si estas especies son representativas para todo el territorio paraguayo, como así también, para determinar la fuente de conocimiento de los vendedores para indicar estas plantas medicinales para 
el tratamiento del estrés y sus afecciones. De las 11 especies citadas como tranquilizante y sedante ${ }^{(20)}$ comercializadas en los mercados de Asunción y la Gran Asunción, solo dos especies son mencionadas en este trabajo y son menta'i y tilo. En las comunidades de Itá Azul y San Gervasio(21), se usan como tranquilizante, cedrón kapi'í, cedrón Paraguay y menta'í, las cuales también se mencionan en este trabajo(22). Se reportan 5 especies de plantas usadas en la atención primaria de la salud, cedrón Paraguay, tilo y toronjil como tranquilizante, mandarina, calmante y sedante y menta'í, para los nervios, coinciden en este trabajo las especies de cedron Paraguay, tilo y menta'í.

\section{CONCLUSIÓN}

En total se reportan 18 especies que se recomiendan para afecciones vinculadas al estrés en los tres mercados y una mezcla que contiene valeriana, flor de tilo, pasionaria, romero.

\section{REFERENCIAS}

1. OMS | Medicina tradicional. WHO [Internet]. 2016 [citado 29 de diciembre de 2020]; Disponible en: http://www.who.int/topics/traditional_medicine/es/

2. WHO. Organización Mundial de la Salud Ginebra Estrategia de la OMS sobre medicina tradicional 2002-2005. OMS [Internet]. 2002 [citado 29 de diciembre de 2020];75. Disponible en: http://apps.who.int/medicinedocs/pdf/s2299s/s2299s.pdf\%0Ahttps:// apps.who.int/iris/bitstream/handle/10665/67314/WHO_EDM_TRM_20 02.1_spa.pdf?sequence $=1$

3. Hsu MC, Creedy D, Moyle W, Venturato L, Tsay SL, Ouyang WC. Use of Complementary and Alternative Medicine among adult patients for depression in Taiwan. J Affect Disord [Internet]. diciembre de 2008 [citado 29 de diciembre de 2020];111(2-3):360-5. Disponible en: https://pubmed.ncbi.nlm.nih.gov/18442859/

4. Hernández M, Novoa M, Civitella S, Masson D, Oviedo A. Plantas usadas en medicina popular en la Isla Santiago, Buenos Aires, Argentina. Boletín Latinoam y del Caribe Plantas Med y Aromáticas. 2013;12(4):985-399.

5. López V, Sueldo B, Franco S, Medina J, Villar López M, Sueldo YB, et al. Conocimiento, aceptación y uso de la medicina tradicional, alternativa y/o complementaria por médicos del seguro social de salud. Rev Peru Med Integr. 2016;1(1):13-21.

6. Bernice L. A medicina tradicional chinesa nos cuidados de saúde em 
Macau. Rev Enferm Ref. 30 de junio de 2017;IV Série(13):157-64.

7. Martinez JL (José L, Muñoz-Acevedo A, Rai M. Ethnobotany : application of medicinal plants. 1.a ed. CRC Press; 2019.

8. Degen RL, Céspedes CI, Gonzáles YP, Gonzáles G, Delmás G, Gonzáles MG, et al. Catálogo ilustrado de 80 plantas medicinales del Paraguay [Internet]. 1.a ed. Ibarrola Diaz D, Degen de Arrua R, editores. Vol. 1. JICA; 2011. 191 p. http://library1.nida.ac.th/termpaper6/sd/2554/19755.pdf

9. Liu L, Liu C, Wang Y, Wang P, Li Y, Li B. Herbal Medicine for Anxiety, Depression and Insomnia. Curr Neuropharmacol [Internet]. 2 de septiembre de 2015 [citado 29 de diciembre de 2020];13(4):481-93. Disponible en: /pmc/articles/PMC4790408/?report=abstract

10. Ravindran A V., Da Silva TL. Complementary and alternative therapies as add-on to pharmacotherapy for mood and anxiety disorders: A systematic review [Internet]. Vol. 150, Journal of Affective Disorders. Elsevier B.V.; 2013 [citado 29 de diciembre de 2020]. p. 707-19. Disponible en: https://pubmed.ncbi.nlm.nih.gov/23769610/

11. Yeung WF, Chung KF, Man-Ki Poon M, Yan-Yee Ho F, Zhang SP, Zhang ZJ, et al. Chinese herbal medicine for insomnia: A systematic review of randomized controlled trials [Internet]. Vol. 16, Sleep Medicine Reviews. W.B. Saunders Ltd; 2012 [citado 29 de diciembre de 2020]. p. 497-507. Disponible en: https://pubmed.ncbi.nlm.nih.gov/22440393/

12. Butler L, Pilkington K. Chinese herbal medicine and depression: The research evidence [Internet]. Vol. 2013, Evidence-based Complementary and Alternative Medicine. Hindawi Limited; 2013 [citado 29 de diciembre de 2020]. p. 14. Disponible en: /pmc/articles/PMC3582075/?report=abstract

13. Mayo Clinic. Síntomas de estrés: consecuencias en tu cuerpo y en tu conducta - Mayo Clinic [Internet]. [citado 29 de diciembre de 2020]. Disponible en: https://www.mayoclinic.org/es-es/healthylifestyle/stress-management/in-depth/stress-symptoms/art-20050987

14. Aguado Martín JI, Bátiz Cano A, Quintana Pérez S. El estrés en personal sanitario hospitalario: estado actual. Med Segur Trab (Madr). 2013;59(231):259-75.

15. McKay DL, Blumberg JB. A review of the bioactivity and potential health benefits of peppermint tea (Mentha piperita L.). Vol. 20, Phytotherapy Research. Phytother Res; 2006. p. 619-33.

16. Akbari F, Rezaei M, Khatony A. Effect of peppermint essence on the pain and anxiety caused by intravenous catheterization in cardiac patients: A randomized controlled trial. J Pain Res. 2019;12:2933-9.

17. Aguilar-Contreras A, Aguilar-Rodríguez S, Xolalpa-Molina S, Politécnico Nacional I. Las verbenaceae empleadas como recurso herbolario en méxico: una revisión etnobotánica-médica the verbenaceae used as an 
herbal resource in mexico: an ethnobotanical-medical review the verbenaceae used as an herbal resource in mexico: an ethnobotanicalme. 2017 [citado 29 de diciembre de 2020];44:195-216. Disponible en: http:www.polibotanica.mx

18. Ragone MI, Sella M, Pastore A, Consolini AE. Sedative and cardiovascular effects of Aloysia citriodora Palau, on mice and rats. Lat Am J Pharm. 2010;29(1):79-86.

19. Así afecta el estrés a tu aparato digestivo. [citado 25 de abril de 2021]; Disponible en: https://www.hola.com/estarbien/20190218137396/como-afecta-estres-aparato-digestivo-cs/

20. Basualdo I, Soria N, Ortiz, M, Degen, R. Plantas Medicinales Comercializadas en los mercados de Asuncion y Gran Asuncion. Rojasiana 2003;6(1):95-114.

21. Degen de Arrua R, Gonzalez, Y. Plantas medicinales utilizadas en las comunidades de Itá Azul y San Gervacio (Paraguay). Revista de Fitoterapia 2014;14 (2):153-166.

22. Soria N, Ramos P. Uso de plantas medicinales en Atencion Primaria de la Salud en Paraguay: algunas consideraciones para su uso seguro y eficaz. Mem. Inst. Investig. Cienc. Salud. 2015;13(2):8-17. 